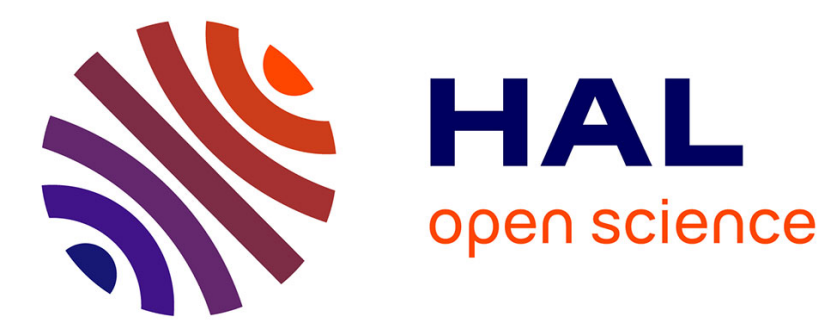

\title{
Correlations and dynamics of polyelectrolyte solutions
}

\author{
J. Hayter, G. Janninck, F. Brochard-Wyart, P.-G. de Gennes
}

\section{To cite this version:}

J. Hayter, G. Janninck, F. Brochard-Wyart, P.-G. de Gennes. Correlations and dynamics of polyelectrolyte solutions. Journal de Physique Lettres, 1980, 41 (18), pp.451-454. 10.1051/jphyslet:019800041018045100 . jpa-00231819

\section{HAL Id: jpa-00231819 https://hal.science/jpa-00231819}

Submitted on 1 Jan 1980

HAL is a multi-disciplinary open access archive for the deposit and dissemination of scientific research documents, whether they are published or not. The documents may come from teaching and research institutions in France or abroad, or from public or private research centers.
L'archive ouverte pluridisciplinaire HAL, est destinée au dépôt et à la diffusion de documents scientifiques de niveau recherche, publiés ou non, émanant des établissements d'enseignement et de recherche français ou étrangers, des laboratoires publics ou privés. 


\title{
Correlations and dynamics of polyelectrolyte solutions
}

\author{
J. Hayter \\ Institut Laue-Langevin, B.P. 156, 38042 Grenoble Cedex, France
}

G. Janninck

SPSRM, CEA, 91190 Gif sur Yvette, France

F. Brochard-Wyart and P. G. de Gennes

Collège de France, 75231 Paris Cedex 05, France

(Reçu le 22 juin 1980, accepté le 22 juillet 1980)

\begin{abstract}
Résumé. - La dynamique des solutions de polyélectrolyte a été étudiée au moyen du diffractomètre à écho de spin pour des vecteurs d'onde $q$ voisins du maximum du facteur de structure statique $\left(q_{\mathrm{m}}\right)$. La dépendance en $q$ du coefficient de diffusion $D(q)$ décroît rapidement pour $q<q_{\mathrm{m}}$, et est à peu près constante pour $q>q_{\mathrm{m}}$. Toutes ces propriétés peuvent être comprises au moyen d'une nouvelle description des corrélations, basée : i) sur la notion d'un trou de corrélation, de diamètre $\xi=a \phi^{-1 / 2}$; ii) des chaînes localement rigides avec une longueur de persistance $b=a \phi^{-1}$ (où $\phi$ est la fraction en volume du polymère et $a$ la taille du monomère).
\end{abstract}

\begin{abstract}
The dynamics of polyelectrolyte solutions have been studied using a neutron spin echo spectrometer for wave vectors $q$ around the maximum of the static structure factor $\left(q_{\mathrm{m}}\right)$. The $q$ dependent diffusion coefficient $D(q)$ decreases sharply below $q=q_{\mathrm{m}}$, and is nearly constant above $q_{\mathrm{m}}$. All these features can be understood in terms of a new picture for the correlations, based on : i) the notion of a correlation hole, of diameter $\xi=a \phi^{-1 / 2}$; ii) locally rigid chains with a persistence length $b=a \phi^{-1}$ (where $\phi$ is the polymer volume fraction and $a$ the monomer size).
\end{abstract}

Polyelectrolyte solutions without salt constitute one of the most mysterious states of condensed matter. Early models represented these solutions as a crystal of rods [1] but the absence of Bragg peaks in the neutron diffraction pattern [2] points towards a liquid state. To improve our understanding of these liquids, we have performed a quasi-elastic neutron scattering study, which probes the motions at frequencies $\omega \sim 10^{8} \mathrm{~s}^{-1}\left(\hbar \omega \sim 5 \times 10^{-8} \mathrm{eV}\right)$ and at wave vectors $q \sim 0.05 \AA^{-1}$. The data show a remarkable variation of the inelastic linewidth, and have led us to a complete reexamination of both static and dynamic scattering functions.

1. Inelastic scattering experiments. - The neutron beam (from the Grenoble high flux reactor, average wavelength $8.3 \AA \pm 8 \%$ ) is scattered by a sample of sulfonated polystyrene $\left(M_{\mathrm{w}}=260.000\right)$, in $\mathrm{D}_{2} \mathrm{O}$, at an SPS concentration $4.78 \%$ by weight. The counter ion is $\mathrm{Na}^{+}$. In a second experiment, sodium bromide 1.5 molar is added to the solution.
The time-dependent correlation function $S(q, t)$ measured by spin echo [3] is roughly exponential, defining a relaxation time $\tau(q)$. We show on figure 1 the effective diffusion coefficient $D(q)=\left(q^{2} \tau(q)\right)^{-1}$ : this is seen to decrease strongly upon increasing $q$ at low $q$, and to become constant at high $q$. The diffusion coefficient with added salt, on the other hand, is nearly constant $\left(7.4 \times 10^{-7} \mathrm{~cm}^{2} / \mathrm{s}\right)$.

2. Static correlations without salt. - The coherent structure factor $S(q)$ has been measured on carefully purified, salt-free SPS solutions [2]. At large $q(q \xi>1)$, where $\xi=a \phi^{-1 / 2}$ is an average distance between neighbouring chains [4] and $a$ the monomer size, the scattering power is typical of a single rod

$$
S(q) \rightarrow S_{\mathrm{s}}(q) \cong(q a)^{-1} .
$$

At smaller $q^{\prime}$ s $(q \xi<1)$, the intensity $S(q)$ drops below. the single rod value $S_{\mathrm{s}}(q)$. There is thus a peak at $q=q_{\mathrm{m}}$, where $q_{\mathrm{m}}(\phi) \sim \phi^{1 / 2}$ from the data of refe- 


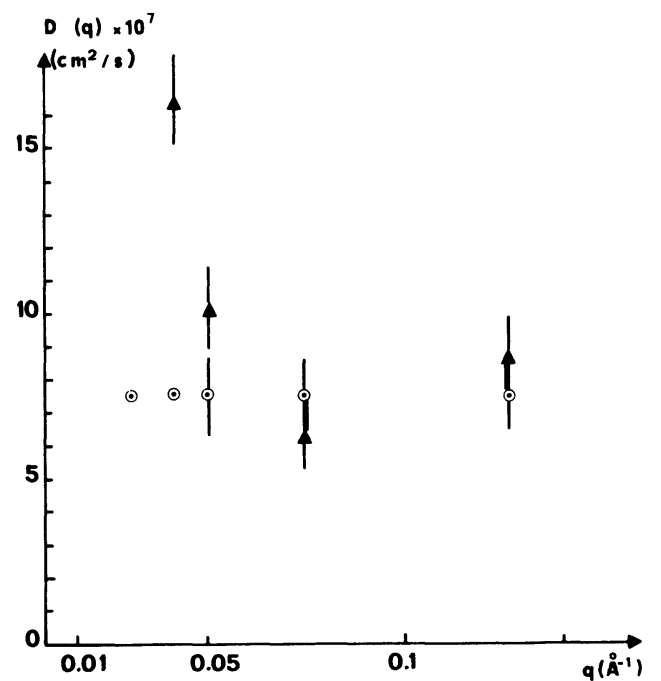

Fig. 1. - Effective diffusion coefficient $D(q)$ for a sulfonated PS solution in $\mathrm{D}_{2} \mathrm{O}$ weight fraction $=4.78 \%$. $\triangle$ : solution without salt ; $\odot$ : solution with $1.5 \mathrm{M} \mathrm{NaBr}$.

rence [2]. We point out however that his peak is not the analog of a short range order peak in a classical liquid where $S\left(q_{\mathrm{m}}\right) \gg S_{\mathrm{s}}\left(q_{\mathrm{m}}\right)$. The present peak is more reminiscent of the correlation hole effect discussed by one of us for polymer melts where each chain carries a labelled region [5]. In both systems the bulk compressibility is very small, and $S(q=0) \ll S\left(q_{\mathrm{m}}\right)$. Each polyelectrolyte chain is surrounded by a correlation tube, from which other chains are strongly expelled. The radius of the tube is the screening length $\kappa^{-1}$; as discussed in reference [4], the screening length scales like the interchain distance in salt-free polyelectrolytes $\kappa^{-1} \cong \xi$. We are thus led to the following form for the correlation function $S_{\mathrm{d}}(q)$ between different chains :

$$
S_{\mathrm{d}}(q)=-S_{\mathrm{s}}(q) \varphi(q \xi) .
$$

Here $\varphi(q \xi)$ is the form factor for the correlation tube.

Incompressibility imposes $\varphi(0)=1$. Also $\varphi(x) \rightarrow 0$ for large $x$. In practice we use $\varphi(x)=\left(1+x^{2}\right)^{-1}$ but the exact form is not important for what follows. Finally :

$$
S(q)=S_{\mathrm{s}}(q) \frac{q^{2} \xi^{2}}{1+q^{2} \xi^{2}}
$$

We must now specify $S_{\mathrm{s}}(q)$ for $q \xi<1$. The authors of reference [4] postulated that, in the isotropic liquid phase, each chain would have a persistence length $b=\xi$. More recently, Odijk proposed a much larger value [6] : $b=\xi^{2} / a=a \phi^{-1}$. He assumed that chain flexibility is controlled entirely by counterion screening. Another contribution to the flexibility comes from random collisions between one chain and its neighbours as shown on figure 2 . To analyse it, we divide each chain into electrical subunits, of length equal to the screening length, each carrying $g=\xi / a$

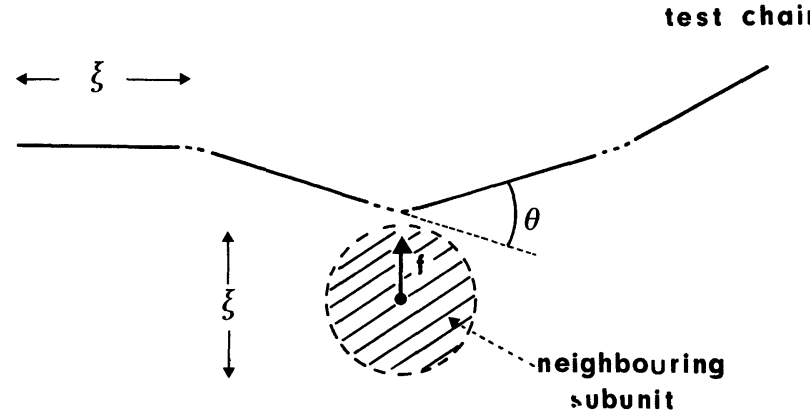

Fig. 2. - One test chain in the polyelectrolyte sees random forces from neighbouring subunits and becomes weakly bent. Inclusion of this effect leads to a persistence length (b) qualitatively similar to the Odijk prediction [6].

monomers. One subunit near the test chain gives a Coulomb force [7] :

$$
f \cong \frac{(g e)^{2}}{\varepsilon \xi^{2}} \cong \frac{k T}{a}
$$

( $e=$ charge per monomer, $\varepsilon=$ dielectric constant. We take $e^{2} / \varepsilon a k T \sim 1$, corresponding to the strong coupling limit). The total force experienced by the subunit on the test chain vanishes, on the average, but the average square does not vanish, because of concentration fluctuations in the surrounding medium.

$$
\left\langle f^{2}\right\rangle \cong\left(\frac{k T}{a}\right)^{2}\left(\frac{\delta \phi}{\phi}\right)^{2} .
$$

Here $\delta \phi$ is the fluctuation of concentration measured in one subunit volume $\xi^{3}$, and is obtained from the osmotic compressibility $\frac{\mathrm{d} \phi}{\mathrm{d} \pi} \sim a^{3} / k T[4]$.

$$
\left\langle\left(\frac{\delta \phi}{\phi}\right)^{2}\right\rangle=\xi^{-3} \frac{k T \mathrm{~d} \phi}{\phi \mathrm{d} \pi} \cong \frac{a}{\xi} .
$$

We now consider the angle $\theta$ between two consecutive units of the test chain, balancing the intrachain Coulomb repulsion between them and the interchain random force $f$. The result is :

$$
\left\langle\theta^{2}\right\rangle=\left\langle f^{2}\right\rangle\left(\frac{a}{k T}\right)^{2} \cong \frac{a}{\xi}
$$

and the associated persistence length $b=\xi /\left\langle\theta^{2}\right\rangle$ agrees with Odijk. Thus we should consider each chain as a sequence of rods each of length $b$ (batonnets). This gives an intrachain structure factor :

$$
S_{\mathrm{s}}(q)=\left\{\begin{array}{ll}
(q a)^{-1} & q b>1 \\
\left(q^{2} b a\right)^{-1} & q b<1
\end{array}\right\} .
$$

Inserting this into eq. (3), we arrive at a total scattering power $S(q)$ with the following features :i) $S(0)=1$ in agreement with osmotic data [4]. ii) In the range $b^{-1}<q<\xi^{-1}, S(q)$ increases linearly with $q$. iii) 
there is a maximum at $q=q_{\mathrm{m}} \cong \xi^{-1}=a^{-1} \phi^{1 / 2}$. iiii) For $q \gg q_{\mathrm{m}}, S(q)$ decreases like $(q a)^{-1}$. All these properties agree rather well with the data of reference [2].

Note that eqs. $(3,8)$ differ strongly from the predictions of a generalized Debye-Hückel theory (Random phase approximation). For instance

$$
q_{\mathrm{m}} a=\phi^{2 / 3}
$$

in RPA. It is not acceptable to treat the large charges (ge) by Debye-Hückel procedures.

We end up this section by some remarks on the existence of an isotropic liquid phase. We have charges $(g e)$ separated by distances $\xi$, and interacting through Coulomb forces which are not strongly screened for such distances. The corresponding coupling constant is $u=(g e)^{2} /(\varepsilon \xi k T)=g$. When $u$ is smaller than a certain characteristic number $u_{\mathrm{c}}(\sim 100)$, we know from computer studies on Coulomb systems that the charges do not make a periodic crystal. Thus the liquid pic phase is favoured when $g<u_{\mathrm{c}}^{+1}$ or $\phi>u_{\mathrm{c}}^{-2}$. At lower concentrations (and with long enough chains) we might have a crystal. Concerning a nematic phase : each batonnet, with it's strong repulsive forces, is sterically equivalent to a hard cylinder, of length $b$, diameter $\xi$, and axial ratio $p=b / \xi \simeq \phi^{-1 / 2}$. For a densely packed system of batonnets, nematic order is expected [9] if $p \widetilde{>}$. This would occur only if $\phi<1 / 64$. Of course these estimates are very rough, but they do give us some explanation for the stability of the isotropic phase.

3. Dynamical effects. - In the $q \rightarrow 0$ limit, we may apply the Kawasaki-Ferrell formula for the diffusion coefficient [10] :

$$
D=S^{-1}(q=0) \int \mathrm{d}_{3} r S(r) \frac{k T}{6 \pi \eta_{\mathrm{s}} r}
$$

where $S(r)$ is the spatial correlation function, and the factor $\left(6 \pi \eta_{\mathrm{s}} r\right)^{-1}$ describes backflow effects in a solvent of viscosity $\eta_{s}$. (More complicated forms incorporating counterion friction can be constructed, but they do not alter the main features.) The function $S(r)$ drops to zero at $r \widetilde{>} \xi$, and is proportional to $r^{-2}$ at small $r$ (rigid rod behaviour). This gives a large diffusion coefficient :

$$
D=\frac{k T}{6 \pi \eta_{\mathrm{s}} a} \ln \left(\frac{\xi}{a}\right) \cong \frac{k T}{6 \pi \eta_{\mathrm{s}} a}
$$

comparable to the diffusion constant for a single monomer. Photon beat data extrapolated to the low salt limit [11] give values in this range $\left(10^{-6}\right.$ to $10^{-5} \mathrm{~cm}^{2} / \mathrm{s}$ ).

At higher $q$ values $(q b>1)$ we may still roughly characterize the dynamics by a $q$-dependent diffusion coefficient [1]

$$
D(q)=k T \frac{\mu(q)}{S(q)}
$$

where $k T S^{-1}(q)$ measures the strength of the restoring forces for a fluctuation $\delta \phi_{q}$ of wave vector $q$, and $\mu(q)$ is the corresponding mobility. Our central remark here is that $\mu(q)$ is not dominated by backflow effects (as it is for neutral, flexible chains), but rather by rigidity effects. A batonnet of length $b$ subjected to forces which are spatially modulated at a wavelength $2 \pi / q$ smaller than $b$, sees a total force which nearly cancels out : only a half wavelength remains uncancelled on the average, and this leads to a reduction factor of order $(q b)^{-1}$. Thus we are led to :

$\mu(q) \cong \mu(0)(q b)^{-1}=\left(6 \pi \eta_{\mathrm{s}} a b q\right)^{-1} \quad(q b>1)$.

Eqs. $(11,12)$ give two regimes : for $b^{-1}<q<\xi^{-1}$, we expect a rapid decrease of the diffusion coefficient :

$$
D(q) \cong D(0)(q b)^{-2}
$$

in qualitative agreement with the spin echo data. For $q \xi>1$, we predict :

$$
D(q)=\frac{D}{g^{2}}=D \phi
$$

independent of $q$, as is observed. We clearly need more measurements to check the power laws (versus $q$ and $\phi$ ) in eqs. $(13,14)$. But on the whole, we conclude that an acceptable description of the isotropic phase of polyelectrolytes without salt can be based on : (1) the Odijk prediction for the rigidity, (2) a correlation hole picture, (3) modified Kawasaki-Ferrell dynamics for rigid rods. With added salt, the properties have not yet been analysed; the coincidence between $D$ values with and without salt at high $q$ may be accidental, but the strong reduction of $D(q=0)$ by salt clearly reflects mainly the known increase of $S(q=0)$ induced by screening [2].

Acknowledgments. - We have benefited from discussions with T. Odijk, P. Pincus, and C. Williams.

\section{References}

[1] Katchalsky, A., Alexandrowicz, Z. and Kedem, O., in Chemical Physics of Ionic Solutions, Connery and Barradas eds. (Wiley, N.Y.) 1966.

[2] Nierlich, M. et al., J. Physique 40 (1979) 701 ;

Williams, C. et al., J. Polym. Sci., Polym. Lett. Ed. 17 (1979) 379; See also ILL report $\mathrm{n}^{\circ}$ 09-01-260, Septembre 1979.

[3] Mezei, F., Z. Phys. 255 (1972) 146.
[4] De Gennes, P. G., Pincus, P., Velasco, R. M. and Brochard, F., J. Physique 37 (1976) 1461.

[5] De GenNes, P. G., Scaling concepts in polymer physics (Cornell U. Press, Ithaca, N.Y.) 1979, p. 64, 65.

[6] Oduj, T., J. Polym. Sci., Polym. Phys. Ed. 15 (1977) 477; Fixman, M., J. Chem. Phys. 70 (1978) 4995 ;

Sxolnick, J. and Fixman, M., Macromolecules 10 (1977) 944. 
[7] Note that screening does not alter eq. (4) because the distance of interest $(\xi)$ is comparable to the screening length $\kappa^{-1}$.

[8] Brush, S., Sahlin, A., Teller, E., J. Chem. Phys. 45 (1966) 2102.

HANSEN, J. P., Molecular Phys. 25 (1973) 1281.

[9] ONSAGER, L., Ann. N. Y. Acad. Sci. 51 (1949) 627 ;

Flory, P., Proc. R. Soc. A 234 (1956) 73.
[10] FerRell, R., Dynamical aspects of critical phenomena edited by J. Budnick and M. Kawatra (Gordon and Breach, New York) p. 1.

See also Hess, W., KleIN, R., Physica 99A (1979) 463.

[11] De Gennes, P. G., Macromolecules 9 (1976) 594. 